CERN - EP/99 - 39

22 March 1999

\title{
A HPMT based set-up to characterize scintillating crystals
}

\author{
C. D'Ambrosio ${ }^{1}$, C. Ercoli ${ }^{1}$, S. Jaaskelainen ${ }^{1}$, G. Lecoeur ${ }^{1}$, H. Leutz ${ }^{1}$, R. Loos ${ }^{1}$, \\ D. Piedigrossi ${ }^{1}$, D. Puertolas ${ }^{2}$, E. Rosso ${ }^{1}$, R. Schomaker ${ }^{3}$ \\ ${ }^{1}$ CERN, Geneva, Switzerland \\ ${ }^{2}$ INFN, Rome, Italy \\ ${ }^{3}$ DEP, Roden, The Netherlands
}

\begin{abstract}
We have developed a fully automatic measurement set-up, capable of measuring light yields arising from scintillating crystals in a linear range of about four orders of magnitude. The photodetector is a Hybrid Photomultiplier Tube especially developed to optimize linear range and photon detection. Crystal and photodetector are temperature controlled by a closed water circuit, as this is essential when measuring low light yield scintillating crystals with a marked temperature dependence of their light yield. Gamma sources can be placed either on top or on the side of the crystal. In this latter case, the source can be automatically moved by a computer-controlled step motor to provide a uniformity profile of the light yield along the crystal. Tagged and not-tagged operation modes are possible. The whole set-up is computer-controlled in an effort to provide fast and reliable measurements, to characterize many crystals per day. This is important for the quality control of the Lead Tungstate crystals that will be applied in the electromagnetic calorimeter of the CMS-detector at the LHC at CERN.
\end{abstract}

To be published in Nuclear Instruments and Methods 


\section{INTRODUCTION}

The Large Hadron Collider (LHC) at CERN will be equipped with two multipurpose detectors, CMS [1] and ATLAS [2]. For CMS, the electromagnetic calorimeter (ECAL) will be composed of Lead Tungstate $\left(\mathrm{PbWO}_{4}\right)$ scintillating crystals [3] read out via Avalanche Photodiodes (APD). The system has to be controlled and monitored in temperature, as the crystal light yield and the APD gain depend on it. The ECAL will contain some 80,000 crystals, with dimensions $\sim 25 \times 25 \mathrm{~mm}^{2}$ (base) tapering down to $21.5 \times 21.5 \mathrm{~mm}^{2}$ (top) along the length of $230 \mathrm{~mm}$. These crystal dimensions will slightly vary with the crystal positions in the calorimeter.

Since a few years, an $R \& D$ effort is going on to characterize the specific properties of $\mathrm{PbWO}_{4}$ crystals and to aim at a crystal growing procedure able to furnish uniform quality crystals. They all have to be measured in size, optically characterized and proven to be radiation hard. In particular, because of a poor scintillation yield and a low light extraction due to the high index of refraction of $\mathrm{PbWO}_{4}(2.16)$, only 40 to 100 photons per $\mathrm{MeV}$ can be extracted on the coupling face to the photon detector. It is essential, therefore, to provide a system, capable of achieving a fast, precise and reliable measurement of $\mathrm{PbWO}_{4}$ light yield.

A few methods [4,5] have been proposed to measure such a poor light yield with a Photomultiplier Tube (PMT) and a ${ }^{60} \mathrm{Co}$ gamma source without having to use an electron accelerator. However, these methods mainly involve deconvolving, via computer simulations, the proper total absorption peak from a noisy and cluttered counting spectrum, generated by $\mathrm{PbWO}_{4}$. The ${ }^{60} \mathrm{Co}$ gammas are emitted at 1.170 and $1.330 \mathrm{keV}$ and, at these energies, Compton scatter interactions amount to almost $70 \%$ of the events. To complicate things, as light yield consists of only a few photoelectrons (5 to 15 photoelectrons in the total absorption peak with the present crystals), all of these signals are smeared by Poisson statistics and therefore all of the contributions are mixed with each other. Finally, single electron resolution (SER) in PMTs is low and limits the recognition of photoelectron peaks in the spectra to the pedestal and the first photoelectron, loading absolute calibration of light yield (photoelectrons detected per $\mathrm{MeV}$ ) with large errors.

Based on these considerations and encouraged by the results obtained in gamma imaging [6,7], we have proposed [8] to apply Hybrid Photomultiplier Tubes (HPMT) [9] to the 
detection of scintillations from gamma excited crystals. To this end, we have developed a specially adapted HPMT in collaboration with DEP $^{1}$ and we have built an automatic setup, insuring temperature stability, measurement reliability and minimum operator time. In the following, we shall describe the experimental set-up, report the first results and show that the measured spectra can be interpreted and reproduced by a simple analytical model.

\section{EXPERIMENTAL SET-UP}

The set-up (Fig. 1) consists of a specially developed HPMT as a photodetector, on which the $\mathrm{PbWO}_{4}$ crystal is placed. The optical contact between the two is ensured by a drop of silicone oil and by the weight of the crystal ( $900 \mathrm{~g})$. The crystal and HPMT are surrounded by a closed-circuit water-cooled envelope, which allows stable and adjustable temperatures during measurements. Outside this envelope and sideways with respect to the crystal longitudinal axis a gamma source $\left({ }^{60} \mathrm{Co}\right.$ or $\left.{ }^{22} \mathrm{Na}\right)$ excites the scintillator. The longitudinal position of this source with respect to the HPMT can be changed in order to measure the uniformity of crystal response. Collinear to the source, a tagging system is installed. It is made of a conical YAP-crystal and a small-diameter photomultiplier tube. The whole set-up is operated under computer control and automated to optimize performance and minimize operator time. In the following the main set-up elements will be discussed in detail.

\section{1 $\mathrm{PbWO}_{4}$ crystals}

In the present phase of development, the size of lead tungstate crystals [1] is $23.8 \times 23.8 \mathrm{~mm}^{2}$ on the base and $20.5 \times 20.5 \mathrm{~mm}^{2}$ on the front face and their length is $230 \mathrm{~mm}$. Specific weight is $8.3 \mathrm{~g} / \mathrm{cm}^{3}$, therefore a crystal weighs $940 \mathrm{~g}$. In the $\mathrm{MeV}$ gamma energy range, absorption properties of $\mathrm{PbWO}_{4}$ can be estimated by properly scaling the lead absorption coefficient curves for photoelectric absorption, Compton scattering and pair production processes. With ${ }^{60} \mathrm{Co}$ excitation, cascade of a $1.17 \mathrm{MeV}$ and a $1.33 \mathrm{MeV}$ gamma, about $30 \%$ of interactions are photoelectric absorption and $70 \%$ are Compton scattering. Since a large fraction of scattered Compton gammas can

\footnotetext{
${ }^{1}$ Delft Electronische Produkten, Roden, The Netherlands
} 
be reabsorbed by the crystal, the fraction of total absorption events can be estimated to be $50 \%$ of the overall counts.

$\mathrm{PbWO}_{4}$ crystals are birefringent. Their emission spectrum ranges between $400 \mathrm{~nm}$ and $650 \mathrm{~nm}$, peaking between 450 and $500 \mathrm{~nm}$, according to the different ions used to dope them $(\mathrm{Nb}, \mathrm{La}$, etc.). Transmission starts typically around $350 \mathrm{~nm}$ and is constant up to $700 \mathrm{~nm}$. Mean refractive index in the emission region is 2.3 [3]. Its light yield is temperature dependent with a $-2 \%$ variation per degree centigrade [10].

\subsection{HPMT}

The working principle of an HPMT has already been described in detail elsewhere [11, 12 and refs therein]. A high resistivity silicon diode faces a suitable photocathode in a vacuum tube. An appropriate electric field accelerates and focuses electrons produced at the photocathode onto the silicon diode, generating electron-hole pairs in a sufficient amount to be detected by proper electronics. The accelerating potential is typically between 10 and $15 \mathrm{kV}$.

The HPMT employed in this study was developed in collaboration with DEP specifically to measure scintillation light from inorganic crystals (Fig 2). The active surface is $40 \mathrm{~mm}$ diameter. A UV-extended S-20 photocathode was evaporated on the $3 \mathrm{~mm}$ thick quartz window. This allows for optimum quantum efficiencies in the near UV-blue spectral region and for optimum light collection efficiency, as the window is flat, thin and wide. The $2 \mathrm{~mm}$ diameter silicon diode produces little electronic noise, shows excellent single photoelectron resolution ( $20 \%$ at FWHM) and resolves peaks with sequentially increasing numbers of photoelectrons up to twenty photoelectrons.

\subsection{Processing electronics, temperature and computer controls}

Signals from the HPMT are first preamplified, then shaped ${ }^{2}$ and stored in a Multi Channel Buffer ${ }^{3}$ (MCB) which is directly computer controlled. This MCB-card allows for triggered or free running data acquisitions. The ADC is peak sensing with 13 bits resolution. The source, either ${ }^{60} \mathrm{Co}(20 \mathrm{kBq})-$ or ${ }^{22} \mathrm{Na}(20 \mathrm{kBq})$, together with the

\footnotetext{
${ }^{2}$ mod. 672 EG\&G Ortec

${ }^{3}$ mod. 926 EG\&G Ortec
} 
tagging unit is shown in Fig. 3. The tagging unit is composed of a truncated conical YAP-crystal (or BGO - crystal) coupled to a Hamamatsu R-1635 photomultiplier tube. Distances between source and $\mathrm{PbWO}_{4}$ crystal, and source and tagging unit respectively can be varied within well-defined limits, to optimize counting rate and tagging efficiency. The source and tagging unit are displaced along the length of the $\mathrm{PbWO}_{4}$ crystal by using a spindle, moved by a fully computer controlled step motor. This allows performing an automatic uniformity scan of the $\mathrm{PbWO}_{4}$ crystal light yield.

Temperature stabilization of the whole unit is achieved with a closed water circuit powered by a cooling unit ${ }^{4}$. It consists of two water-cooled copper boxes, one very close to the crystal (Fig. 1) and another hosting the whole mechanical part of the set-up. A foam and wood box provides the thermal insulation from the environment. Crystal temperature is controlled and stabilized by temperature probes in a feedback loop [10].

The full set-up operation is integrated and controlled by a specially developed Labview ${ }^{5}$ program together with a computer interfacing card $^{6}$.

\section{MEASUREMENTS AND RESULTS}

\section{$3.1{ }^{60} \mathrm{Co}$ - measurement (tagging off)}

At the present production performance, the resulting $\mathrm{PbWO}_{4}$ crystals produce between 5 and 15 photoelectrons per $\mathrm{MeV}$ photon energy as measured by the multialkali photocathode of the HPMT. As Compton processes can amount to as much as $70 \%$ of the counted events, the main task when analyzing ${ }^{60} \mathrm{Co}$-spectra obtained with $\mathrm{PbWO}_{4}$ crystals is to extract the total absorption peak from the spectrum and to find the average light yield in terms of photoelectrons per MeV. In Fig. 4, a spectrum of $\mathrm{PbWO}_{4}{ }^{60} \mathrm{Co}-$ scintillations (crystal \#1494) is shown. A first evident advantage of this HPMT system as compared with standard photomultipliers is the presence of photoelectron peaks, which allows for direct and absolute calibration. Gain stability and reproducibility can be therefore controlled on line, saving measurement time and improving precision dramatically. In Fig. 4, pedestal, photoelectron peak one and peak two have been omitted in order to minimize electronics dead time and low energy (Compton,

\footnotetext{
${ }^{4}$ Lauda, mod. R6

${ }^{5}$ National Instruments

${ }^{6}$ mod. PC+, National Instruments
} 
backscatters, etc.) noise. This could not have been done with a photomultiplier tube, as pedestal position and first photoelectron peak position (the only clearly visible) have to be known precisely.

A uniformity scan consists of moving the ${ }^{60}$ Co source along the crystal axis, taking a spectrum for each position and analyzing it to get the related number of photoelectrons per $\mathrm{MeV}$. Spectra measurements are performed automatically by a computer routine ${ }^{7}$ controlled in turn by the Labview program that supervises the whole scan.

It is possible to choose two types of on-line analysis. The first consists in subtracting the background (Compton) spectrum which is defined by a Region-Of-Interest (ROI) file $^{8}$ and then fitting the remaining spectrum with a Gaussian distribution. Peak and width of the Gaussian are then stored. This works well for crystals emitting more than $\sim 10$ photoelectrons per $\mathrm{MeV}$, as the separation between the Compton and photoelectric contributions is sufficiently well-resolved. For smaller photoelectron yields and for tagged measurements (see section 3.2), a fit of the entire spectrum is required. The source is then automatically moved to the next position and the data acquisition starts again. According to the count rates and the required precision, an entire scan along the crystal at 21 positions can take as little as 6 minutes with no operator intervention in between. Most of the time is taken to install the crystal on the HPMT and to wait until the chosen temperature is attained.

In Fig. 5, a ${ }^{60} \mathrm{Co}-\mathrm{scan}$ of $\mathrm{PbWO}_{4}$ crystal \#1858 is shown. This curve shows a parabolalike behaviour: moving the source away from the HPMT, the emitted light suffers increasing absorption losses in the crystal, therefore the light yield decreases. On the other hand, moving the source further away, a larger fraction of light can overcome the total reflection angle cut-off, due to the geometrical shape of the crystals that tends to decrease light incidence angles on the crystal-HPMT interface. Very transparent crystals will show an almost monotonic increase of the light yield versus increasing distance to the HPMT.

In Fig. $6{ }^{60} \mathrm{Co}$-scans of $\mathrm{PbWO}_{4}$ crystal \#1494 are shown with pulse shaping varying from $0.1 \mu$ s to $2 \mu$ s. These results clearly show a small loss due to the light decay time of $\mathrm{PbWO}_{4} .90 \%$ of the light is collected in $0.1 \mu \mathrm{s}$, indicating a decay time around $30 \mathrm{~ns}$ for this crystal.

\footnotetext{
${ }^{7}$ Maestro, EG\&G Ortec

${ }^{8}$ A Region Of Interest defines the spectrum interval of interest for the analysis
} 
For the calorimetric application, it is essential to measure the slope of the scan curve (e.g., Figs 5 and 6) in the $\sim 100 \mathrm{~mm}$ furthest from the HPMT. This region represents roughly 5 times the anticipated radiation length $X_{0}$, where most $(>99 \%)$ of the electromagnetic shower takes place (see Table 1). If the slope of the scan curve is too large, the uniformity characteristics of the crystal will have to be modified to obtain a slope smaller than $0.35 \%$ per $\mathrm{X}_{0}$ of $\mathrm{PbWO}_{4}$. Therefore, the error of the slope measurement must be kept small and, from a statistical analysis of many crystals measured, we estimated a slope error of $\sim 0.2 \%$ per $\mathrm{X}_{0}$ for the set-up.

\section{$3.2{ }^{22}$ Na-measurement (tagging on)}

${ }^{22} \mathrm{Na}$ is a positron emitter with end point energy of $0.54 \mathrm{MeV}$. The positron emission is followed by a $1.275 \mathrm{MeV}$ gamma emission. Coming to rest within the source, the positron annihilates with an electron thus emitting two collinear $0.511 \mathrm{MeV}$ photons. The principle of the ${ }^{22} \mathrm{Na}$ measurements is to tag one $0.511 \mathrm{MeV}$ photon, in order to measure scintillations generated by the second $0.511 \mathrm{MeV}$ photon in $\mathrm{PbWO}_{4}$. Care has been taken to avoid simultaneous measurements of the $0.511 \mathrm{MeV}$ annihilation photons and the $1.275 \mathrm{MeV}$ photons. A certain distance from the $\mathrm{PbWO}_{4}$ surface is necessary to minimize this effect. However, the source cannot be too far from the crystal in order to get a good counting rate and to avoid edge effects. Two ${ }^{22} \mathrm{Na}$ spectra taken with tagging on and off are shown in Fig. 7 for a $\mathrm{PbWO}_{4}$ crystal. The pedestal peak is omitted for both. The improvement in total absorption peak visibility (the peak generated by totally absorbed $0.511 \mathrm{MeV}$ photons) is evident and the resulting spectra can be described by a simple model (see section 4).

A critical aspect with the tagging technique is the choice of the tagging crystal. In our case we mounted a small YAP crystal with high light yield, fast decay time but a very small total absorption cross-section compared with the Compton cross-section, see Table 1 and Fig. 3. We ruled out the use of a BGO crystal because of the poor light yield and slow decay time.

As the number of photoelectrons achievable with a $0.511 \mathrm{MeV}$ gamma in $\mathrm{PbWO}_{4}$ is between 3 to 8 , a complete fit of the resulting spectra is needed. This will be the subject of the next section together with a general discussion of the results. In Fig. 8, two ${ }^{22} \mathrm{Na}$ tagged spectra measured at different temperatures are shown. 


\section{DISCUSSION}

The ${ }^{22} \mathrm{Na}$ spectra in Fig. 8 show a marked discrete structure superimposed on a small continuum. This distribution is nearly Poissonian and each Gaussian peak represents a photoelectron number, with its normalized area $\mathrm{P}_{\mathrm{i}}$ equal to

$$
P_{i}=\frac{(\mu)^{i}}{i !} e^{-\mu} \quad \text { and } \quad \sum P_{i}=1
$$

where $\mu$ is the average number of photoelectrons and $i$ the actual number of detected photoelectrons. The width of these Gaussian peaks is set by the preamplifier electronic noise added in quadrature with the equivalent capacitive diode noise. As discussed in previous papers (see for example [11]), the diode noise is much smaller than the preamplifier noise. The total noise will therefore not depend in first approximation on the square root of $i$ as it does in the case of photomultiplier tubes.

The deviation from a proper Poissonian distribution, characterized by the small continuum, visible between and under the peaks, can be fully accounted for by the backscattering of photoelectrons from the silicon diode. In fact, there is a finite probability $(\sim 20 \%)$ that a $15 \mathrm{keV}$ photoelectron is backscattered from the silicon diode surface. In doing so, the photoelectron leaves on average about $60 \%$ of its energy in the diode, with a distribution that spans between 0 and $100 \%$ of the photoelectron energy [13].

To fit the experimental spectra in Fig 8, let us start with a first approximation of the "intrinsic" $\mathrm{PbWO}_{4}$ scintillation spectrum due to ${ }^{22} \mathrm{Na} 511 \mathrm{keV}$ gamma excitation. This can be roughly approximated by the distribution in Fig. 9.

Every time the $511 \mathrm{keV}$ gamma energy is totally absorbed, $\left\langle\mathrm{n}_{\gamma}\right\rangle$ photoelectrons will be produced from the photocathode on average. Compton scattering on the other hand is responsible for the continuum that starts at zero and ends at the Compton edge energy, given by

$<n_{c}>=\frac{<n_{\gamma}>}{1+m_{0} c^{2} / 2 E_{\gamma}}$

As $\left\langle\mathrm{n}_{\gamma}\right\rangle$ in $\mathrm{PbWO}_{4}$ is small, the intensity distribution of measured photoelectrons follows a Poissonian distribution given by Eq. 1 for the total absorption peak, 
$P_{i}\left(<n_{\gamma}>\right)=\frac{\left(<n_{\gamma}>\right)^{i}}{i !} e^{\left.-<n_{\gamma}\right\rangle}$

where $\mathrm{P}_{\mathrm{i}}\left(\left\langle\mathrm{n}_{\gamma}\right\rangle\right)$ gives the probability of measuring $i$ photoelectrons.

To obtain the equivalent expression for the Compton distribution, we have to convolve the square distribution $\mathrm{C}(\mathrm{x})$ in Fig. 9 with the Poissonian distribution, that is

$P_{c_{i}}\left(<n_{c}>\right)=\frac{1}{f_{c}} \int_{0}^{<n_{c}>} C(x) P_{i}(x) d x \quad$ where $\quad f_{c}=\int_{0}^{<n_{c}>} C(x) d x$

which is still $\quad \sum P_{c_{i}}\left(<n_{c}>\right)=1$

Eq. 4 can be solved to give

$P_{c_{i}}\left(<n_{c}>\right)=P_{c_{i-1}}\left(<n_{c}>\right)-\frac{P_{i}\left(<n_{c}>\right)}{<n_{c}>} \quad$ and $\quad P_{c_{0}}\left(<n_{c}>\right)=\frac{1-e^{-<n_{c}>}}{<n_{c}>}$

with $\quad P_{i}\left(<n_{c}>\right)=\frac{\left(<n_{c}>\right)^{i}}{i !} e^{\left.-<n_{c}\right\rangle} \quad$ being the Poisson distribution for $\left\langle\mathrm{n}_{\mathrm{c}}\right\rangle$.

The resulting spectrum is the weighted sum of total absorption events and Compton events,

$P_{T_{i}}=\left(1-f_{c}\right) P_{i}\left(<n_{\gamma}>\right)+f_{c} P_{c_{i}}\left(<n_{c}>\right)$ for all $i$

Here, $\left\langle\mathrm{n}_{\gamma}\right\rangle$ is the average number of photoelectrons produced on the photocathode of the HPMT. To obtain now the measured spectrum, we have to again convolve Eq. 3 and the set of Eqs 5, first with the backscattering distribution and then with the preamplifier electronic noise shaping function. As we essentially follow equations 5 through 7 in reference [11] for these two steps, we refer to that paper for a detailed discussion. Finally, the result has to be added together as in Eq. 6, where $\mathrm{P}_{\mathrm{i}}\left(\left\langle\mathrm{n}_{\gamma}\right\rangle\right)$ and $\mathrm{Pc}_{\mathrm{i}}\left(\left\langle\mathrm{n}_{\gamma}\right\rangle\right)$ are now the product of three convolution steps.

The spectrum resulting from all the previous transformations is shown in Fig. 10, together with its total absorption and Compton continuum contributions. It is worth noting the effect of Poisson statistics on the sharp edge of the Compton distribution in Fig. 9, which is now completely smeared out. In Figs 11a,b, comparisons between a 
measured spectrum and a calculated spectrum for tagged ${ }^{22} \mathrm{Na}$ excitation and for ${ }^{60} \mathrm{Co}$ excitation are shown respectively. Due to the backscattering process, the average number of measured photoelectrons is lower by a factor $\sim 0.85$ from the average number of photoelectrons produced at the photocathode (compare Figs 4 and 11b). Note also that for the ${ }^{22} \mathrm{Na}$ spectrum, the Compton fraction of events is only $\sim 25 \%$, but the two contributions overlap considerably. On the contrary, in the case of ${ }^{60} \mathrm{Co}$ excitation, the resulting Compton fraction is $\sim 50 \%$, but the total absorption peak is at double the photoelectron number. In Fig. 12, the ratio between ${ }^{60} \mathrm{Co}$ and ${ }^{22} \mathrm{Na}$ scans is shown. It shows the good agreement between the two experimental methods. However, for crystals with a light yield larger than $\sim 10$ photoelectrons per $\mathrm{MeV},{ }^{60} \mathrm{Co}$ measurements are easier and much faster to perform. For lower light yield values ${ }^{22} \mathrm{Na}$ tagged measurements will be necessary to achieve the required slope precision. 


\section{CONCLUSIONS}

We have developed and operated a new set-up, which measures light yields from scintillating crystals. Based on a specially developed HPMT, completely automatic and temperature controlled, this set-up features fast, reliable and precise scintillation measurements. Gain stability and reproducibility can be controlled on line, saving on measurement time and improving precision. In particular, this set-up was constructed to characterize the scintillation performance of $\mathrm{PbWO}_{4}$ crystals, as they will be used in the electro-magnetic calorimeter of the CMS detector at CERN. It provides two modes of operation (tagged and not tagged), which prove to be useful when the crystal light yield is lower or larger than 10 photoelectrons per $\mathrm{MeV}$, respectively. The evaluation of the crystal light yield can be achieved with a fitting routine based on a simple analytical model and, therefore, it can be performed on-line and in a very short time (a few seconds) even with a small personal computer.

\section{ACKNOWLEDGEMENTS}

This work was an R\&D project carried out by the EP-TA2 group at CERN. $\mathrm{PbWO}_{4}$ crystals were kindly handed to us by EP-CMA group and were grown at the Bogoroditsk Technological Plant in Russia. Partial financial support from the EP-CMA group at CERN is acknowledged. Finally, we thank A. Peyaud and G. Lefebvre for their help and P. Lecoq for the careful reading of the manuscript. 


\section{REFERENCES}

[1] The CMS Collaboration, "The Compact Muon Solenoid", Technical Proposal, CERN/LHCC 94-38 (1994)

[2] The ATLAS Collaboration, "ATLAS, Technical proposal for a general purpose pp experiment at the large hadron collider at CERN", Technical Proposal, CERN/LHCC 94-43 (1994)

[3] The CMS Collaboration, "The Electromagnetic Calorimeter Project", Technical Design Report, CERN/LHCC 97-33, 15 December 1997

[4] S. Baccaro et al., "Precise determination of the light yield of scintillating crystals", Nucl. Instr. and Meth. , vol. A 385, p. 69-73, (1997)

[5] J. Wu et al., "The precise determination of the light yield of the $\mathrm{PbWO}_{4}$ crystal", Nucl. Instr. and Meth. , vol. A 404, p. 311-314, (1998)

[6] D. Puertolas, D. Piedigrossi, R. Pani, H. Leutz, T. Gys, F. De Notaristefani, C. D'Ambrosio, “An ISPA-camera for $\gamma$-rays", I.E.E.E. Trans. Nucl. Sci., vol. 42, 1995, p. 2221-2228.

[7] D. Puertolas, D. Piedigrossi, H. Leutz, T. Gys, F. De Notaristefani, C. D'Ambrosio, "An ISPA-camera for $\gamma$-rays with improved energy resolution", I.E.E.E. Trans. Nucl. Sci., vol. 44, 1997, p. 1747-1752.

[8] C. D'Ambrosio, "First results on crystal light yield measurements with a Hybrid Photo Multiplier Tube”, CERN, Min ECAL/PWO/LY-01, MS, 21 June 1995

[9] C. D'Ambrosio et al., "The ISPA-tube and the HPMT, two examples of a new class of photodetectors: the Hybrid Photo Detectors“, Nuclear Physics B (Proc. Suppl.) 61B (1998) 638-643.

[10] C. D'Ambrosio, C. Ercoli, S. Jaaskelainen, E. Rosso, P. Wicht, Nucl. Instr. and Meth. , vol. A 388, p. 119-283, (1997)

[11] C. D'Ambrosio, T. Gys, H. Leutz, D. Piedigrossi, D. Puertolas, S. Tailhardat, Nucl. Instr. and Meth., vol. A 338, p. 389-397, (1994)

[12] C. D'Ambrosio, T. Gys, H. Leutz, D. Piedigrossi, D. Puertolas, S. Tailhardat, T. Shimizu, O. Shinji, Nucl. Instr. and Meth. , vol. A 345, p. 279-283, (1994)

[13] E. H. Darlington, J. Phys. D: Appl. Phys, vol. 8, p. 85-93, (1975) 
Table 1: Properties of several inorganic crystal scintillators [3]

\begin{tabular}{|l|l|l|l|l|l|l|}
\hline Material & $\mathrm{NaI}(\mathrm{Tl})$ & $\mathrm{CsI}$ & $\mathrm{BGO}$ & $\mathrm{PbWO}_{4}$ & $\mathrm{YAP}(\mathrm{Ce})$ & $\mathrm{CeF}_{3}$ \\
\hline Density $\left[\mathrm{g} / \mathrm{cm}^{3}\right]$ & 3.67 & 4.51 & 7.13 & 8.28 & 5.37 & 6.16 \\
\hline Radiation length $\mathrm{X}_{0}[\mathrm{~cm}]$ & 2.59 & 1.85 & 1.12 & 0.89 & 2.7 & 1.68 \\
\hline Light decay time $[\mathrm{ns}]$ & 230 & 16 & 300 & 15 & 27 & 25 \\
\hline Refractive index & 1.85 & 1.80 & 2.20 & 2.16 & 1.95 & 1.62 \\
\hline Maximum of emission $[\mathrm{nm}]$ & 410 & 315 & 480 & 440 & 365 & 300,340 \\
\hline Temperature coeff. $\left[\% /{ }^{\circ} \mathrm{C}\right]$ & $\sim 0$ & -0.6 & -1.6 & -2 & $\sim 0$ & 0.14 \\
\hline Relative light output & 100 & 20 & 15 & 1.3 & 40 & 8 \\
\hline
\end{tabular}




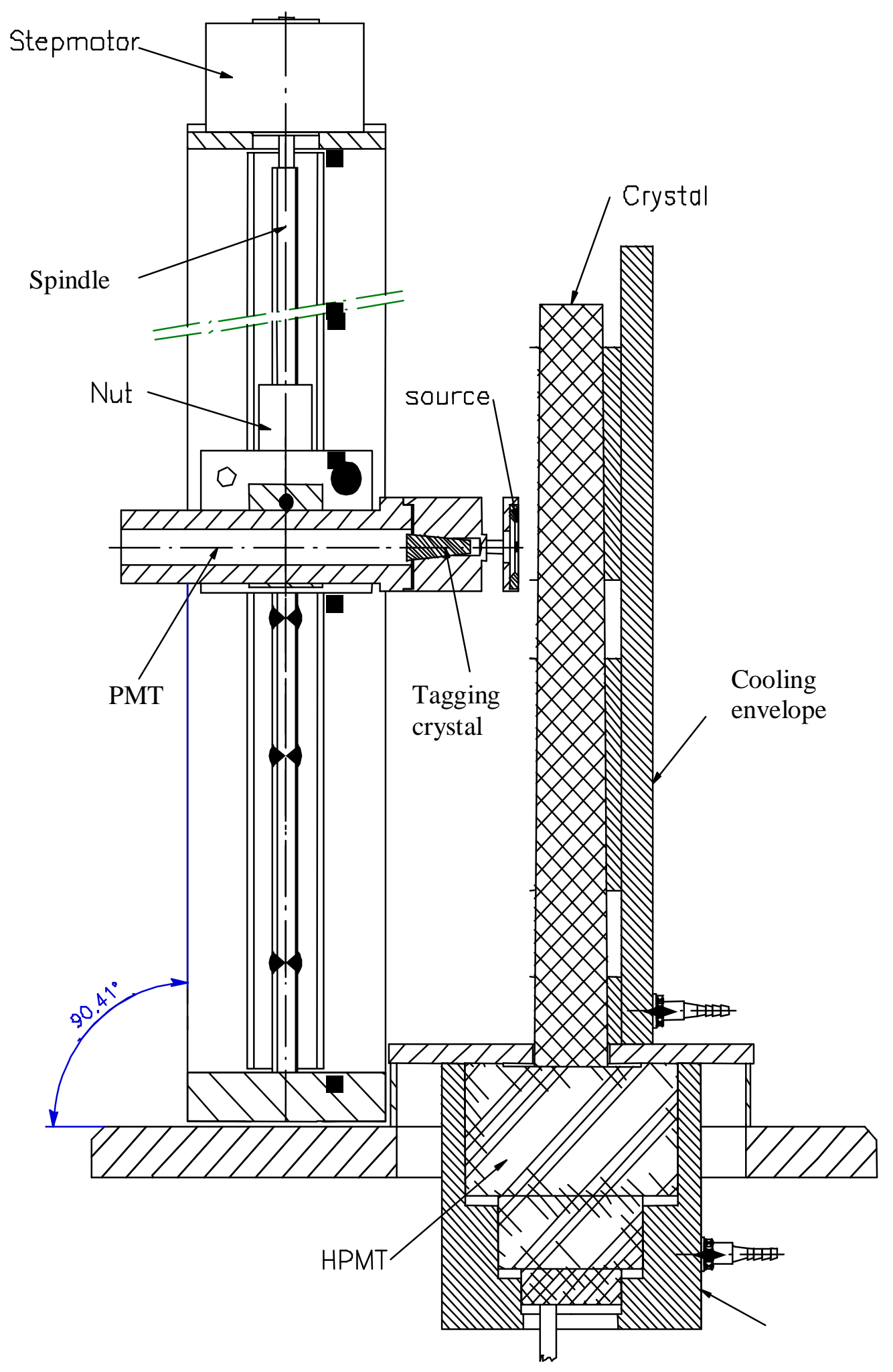

Fig. 1: Components of the set-up 
$3 \mathrm{~mm}$ thin quartz window

$40 \mathrm{~mm}$ diameter photocathode

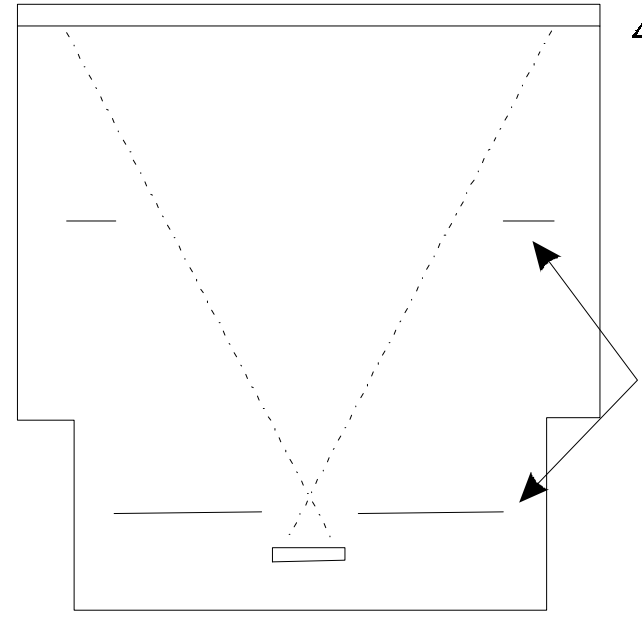

vacuum sealed envelope

focussing electrodes

diode

accelerating potential between $5 \mathrm{kV}$ and $15 \mathrm{kV}$

Fig. 2 Thin quartz window HPMT (mod. PPO 470 A) 


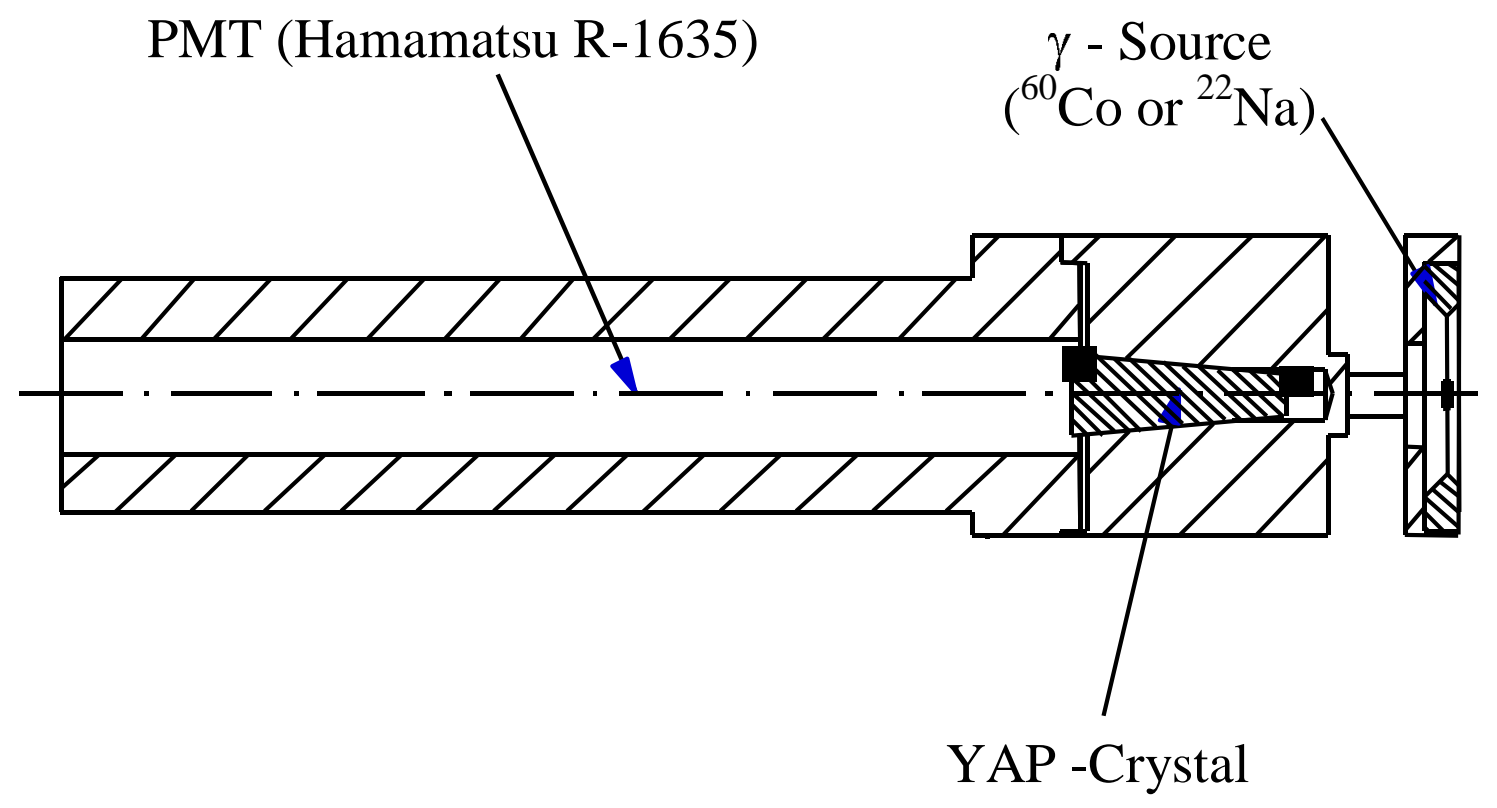

Fig 3: Tagging unit layout. 
- 17 -

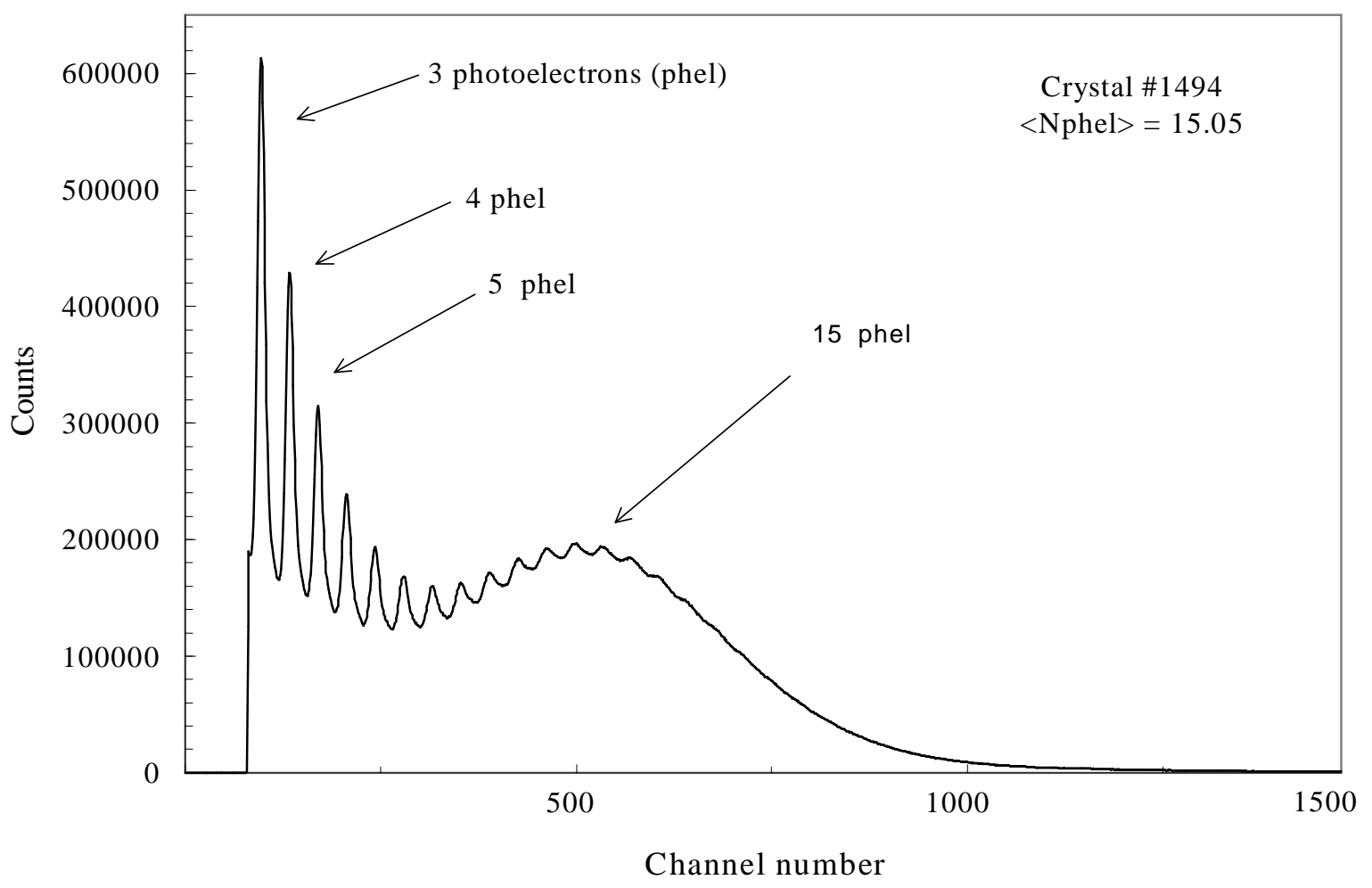

Fig. 4: ${ }^{60}$ Co spectrum, measured using $\mathrm{PbWO}_{4}$ crystal \# 1494. 


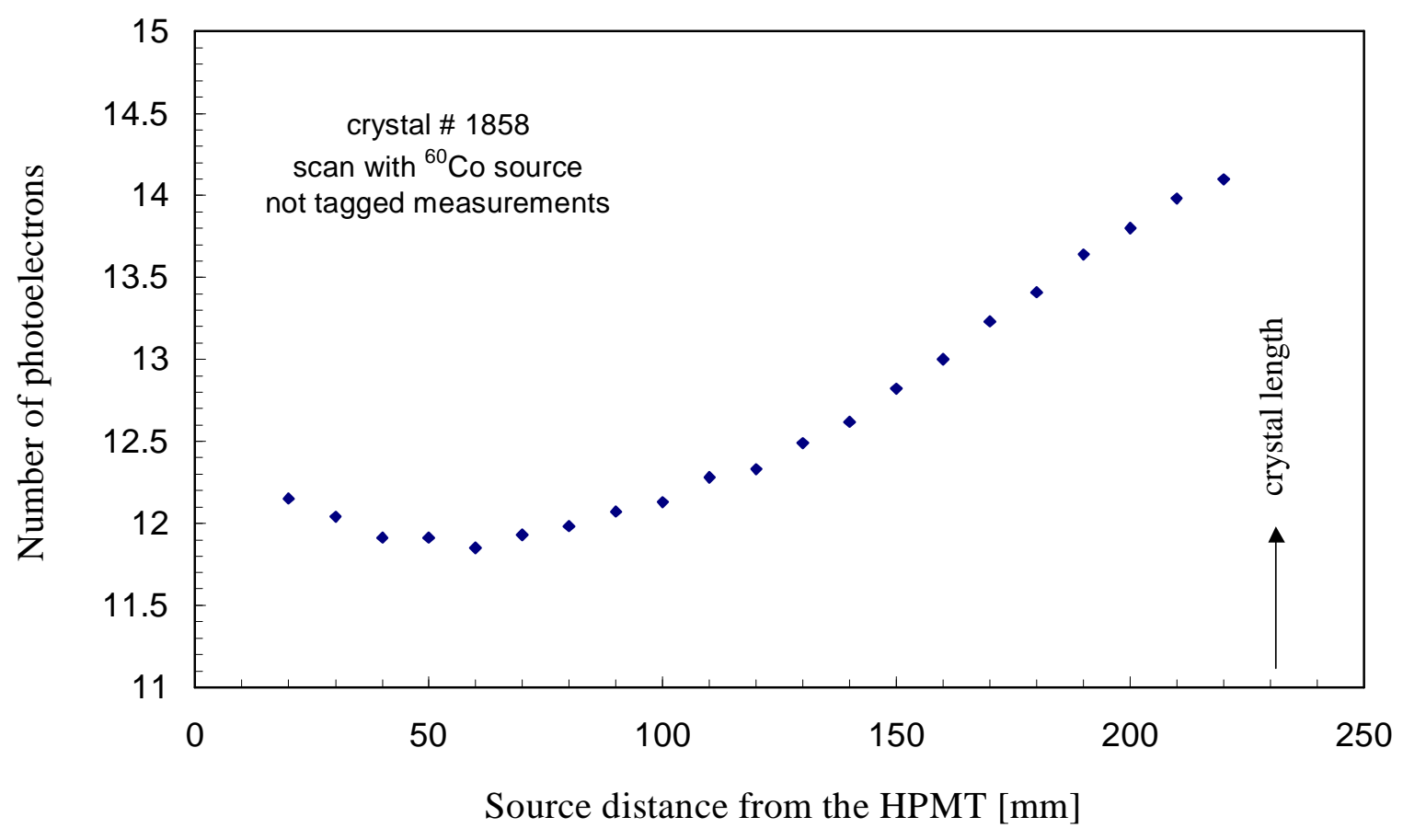

Fig. 5: Scan of $\mathrm{PbWO}_{4}$ crystal \#1858 at different excitation positions with laterally arranged ${ }^{60} \mathrm{Co}$ source. The crystal response in photoelectrons at the total absorption peak is represented as a function of the source distance from the HPMT. 


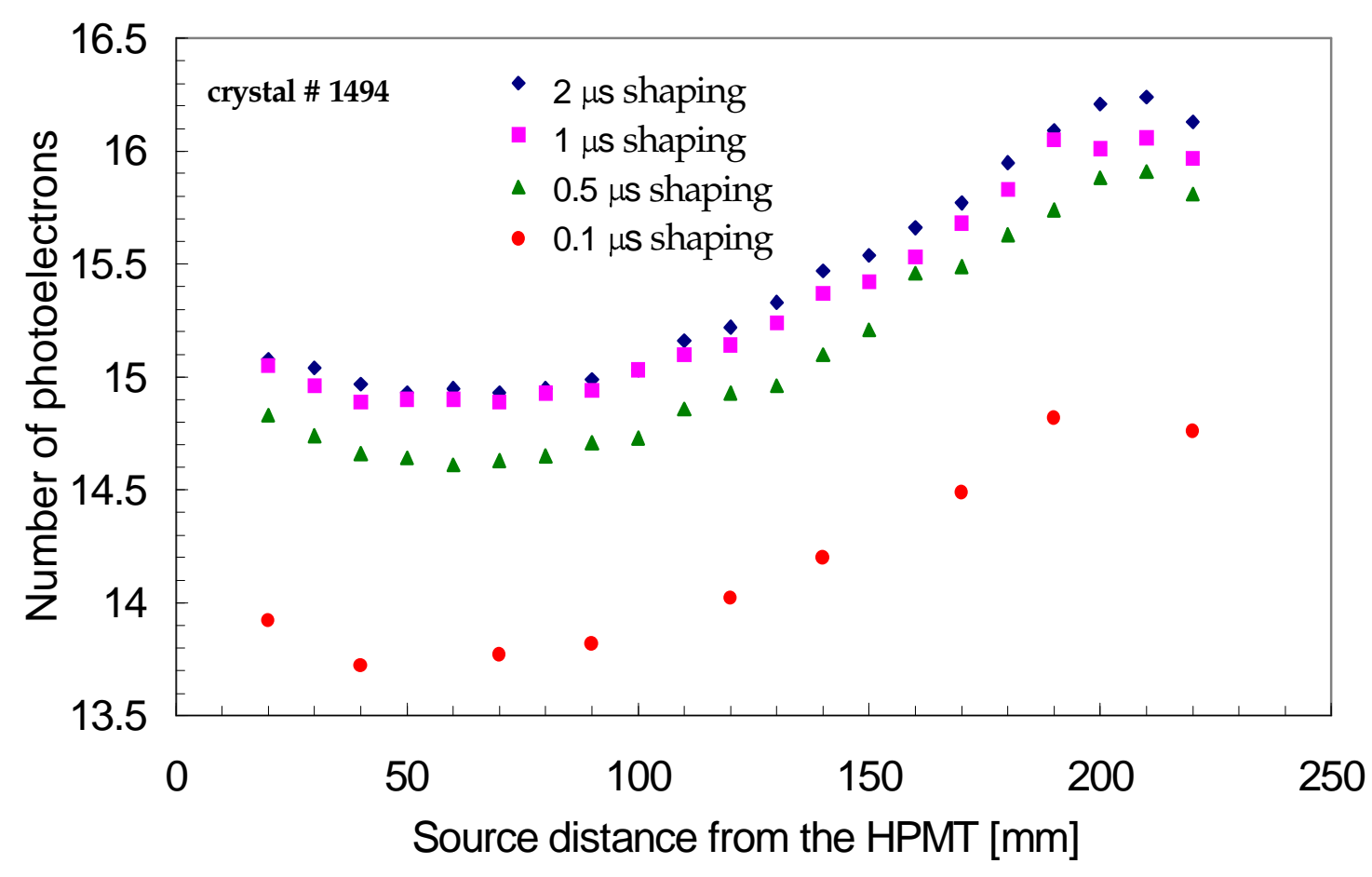

Fig. 6: Non-uniformity plots of $\mathrm{PbWO}_{4}$ crystal \#1494, realised at different shaping times. 


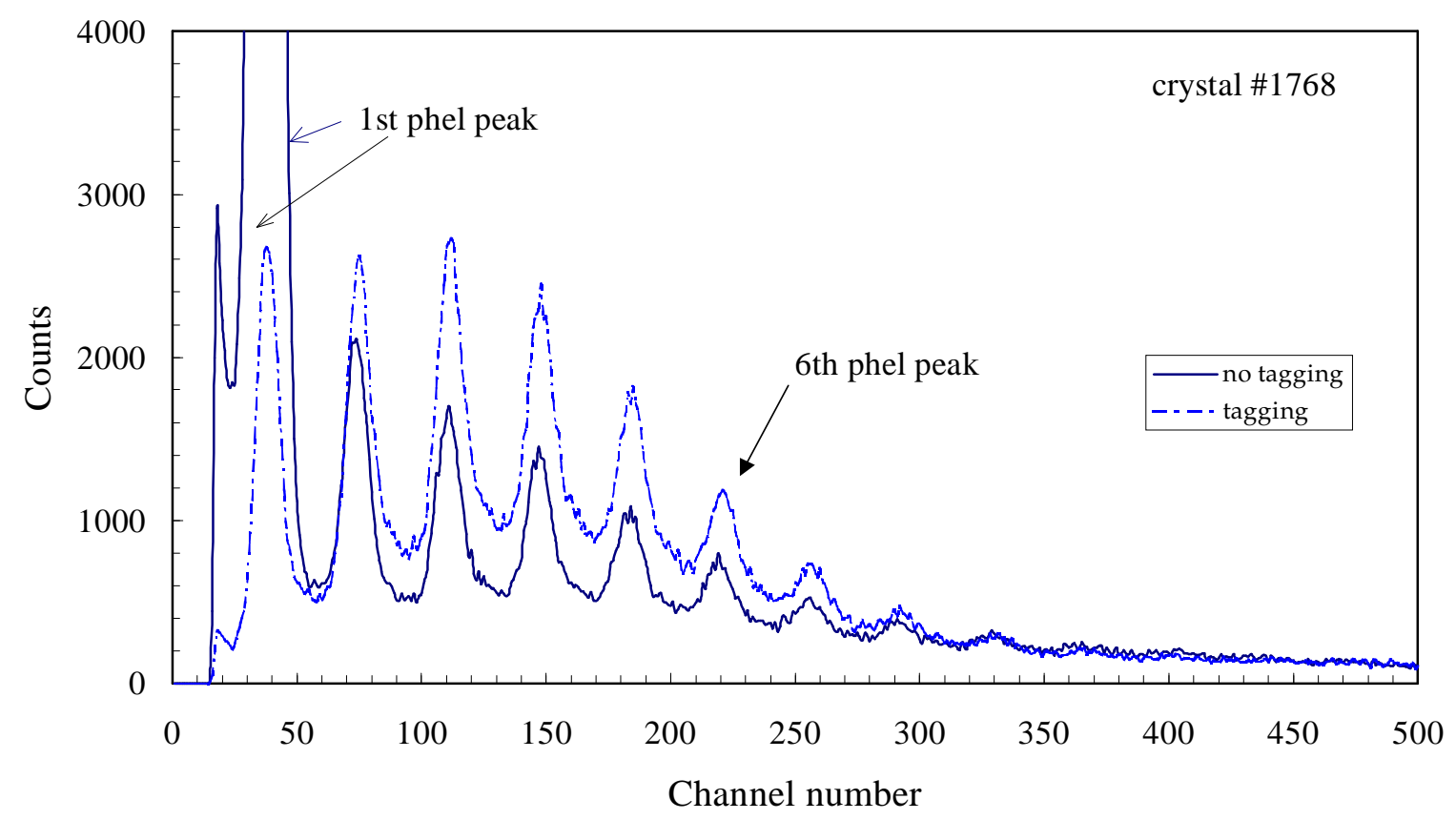

Fig. 7: ${ }^{22} \mathrm{Na}$ spectra realised with tagging off (continuous line) and tagging on (dashed line). Counting rates are different between tagged and not tagged spectra (depending on the geometrical arrangement, between 10 and 20 times lower counting rates for tagged measurements have to be expected). Therefore, the respective acquisitions have been stopped in order to equal the counts of the spectra between channels 300 and 500. In spite of the much lower counting rate, the tagged spectrum shows no background counts at low energies, contrary to the non tagged spectrum, where most of the counts are background-related (especially for the first and second phel. peaks). 


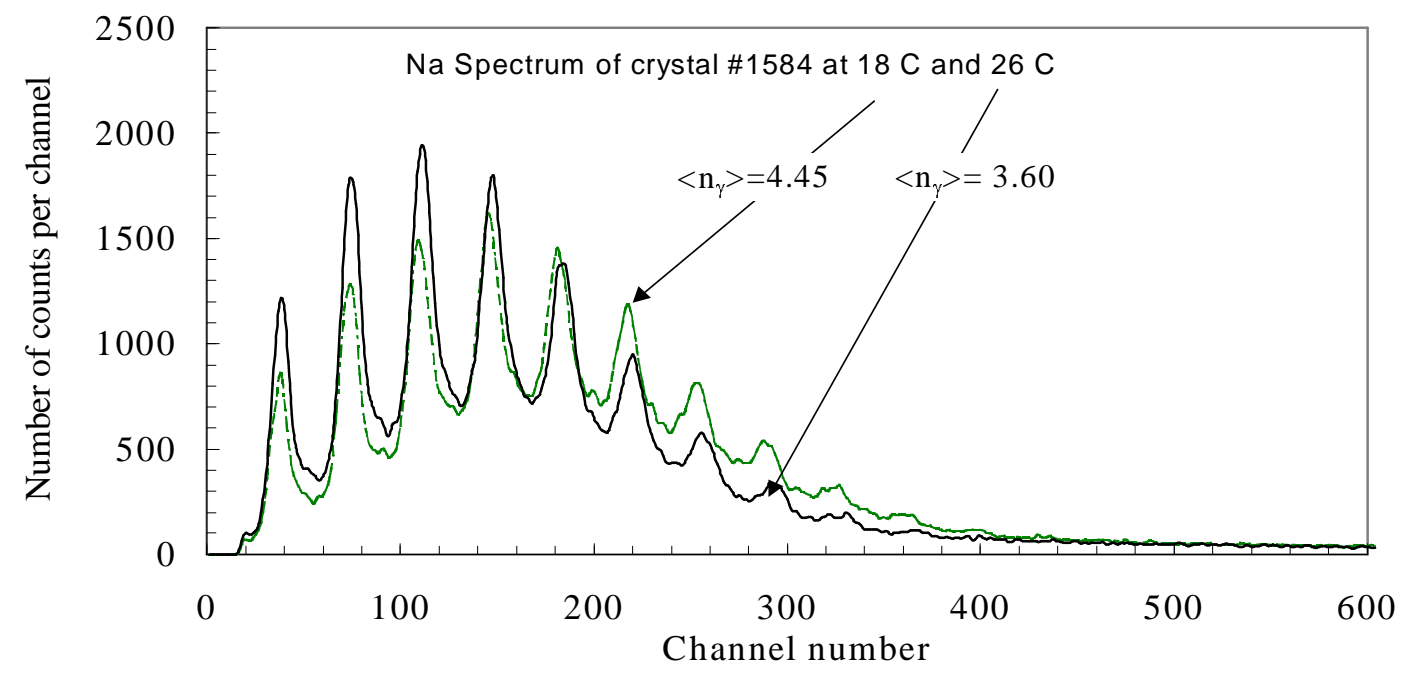

Fig. 8: ${ }^{22} \mathrm{Na}$ spectrum realized with $\mathrm{PbWO}_{4}$ crystal \#1584 at different temperatures $\left(14^{\circ} \mathrm{C}\right.$ and $\left.26^{\circ} \mathrm{C}\right)$; the light yield decreases with increasing temperature at a rate of $\sim 2 \%$ per ${ }^{\circ} \mathrm{C}$ giving an average of 4.45 photoelectrons at $18{ }^{\circ} \mathrm{C}$ and 3.60 at $26{ }^{\circ} \mathrm{C}$.

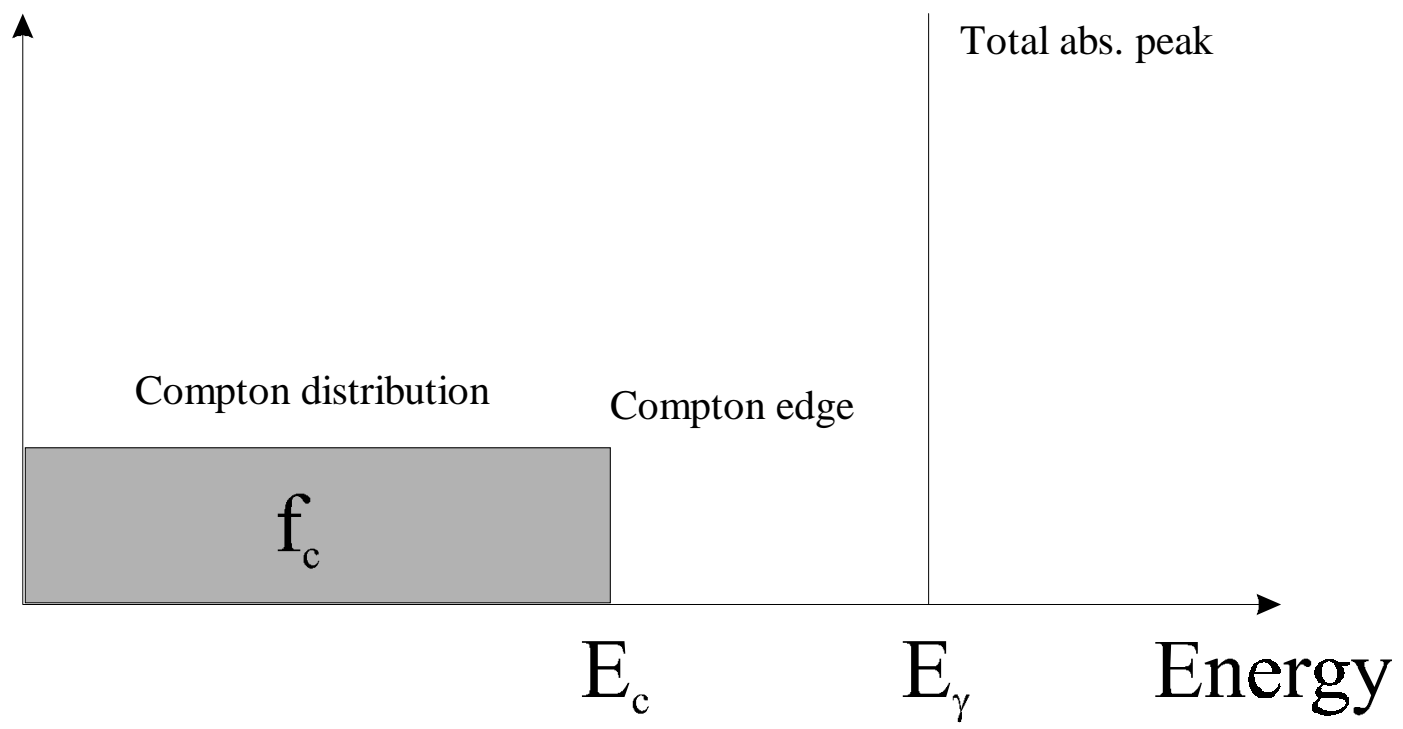

Fig. 9: Approximated energy spectrum of the interaction in $\mathrm{PbWO}_{4}$ of ${ }^{22} \mathrm{Na} 511 \mathrm{keV}$ photons. 


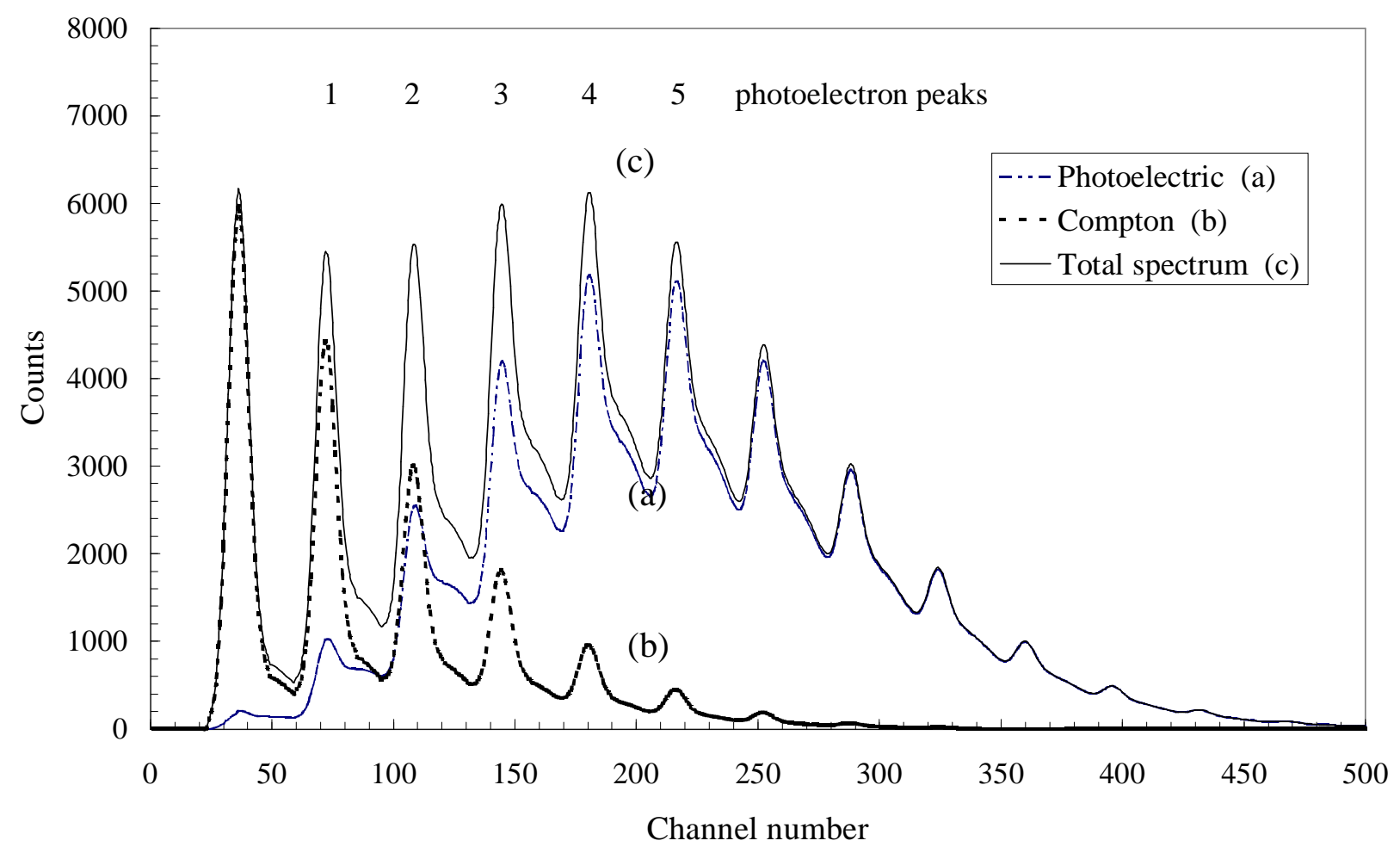

Fig. 10: Example of ${ }^{22} \mathrm{Na}$ spectrum simulation. Note the calculated energy distribution components - Compton (a) and photoelectric (b) - which have been taken into account in the program and their sum (c) (Eq.6). The photoelectric fraction of events is 0.75 and the average number of photoelectrons $\left\langle\mathrm{n}_{\gamma}\right\rangle$ is $6.0 .\left\langle\mathrm{n}_{\mathrm{c}}\right\rangle=2 / 3\left\langle\mathrm{n}_{\gamma}\right\rangle$ is calculated from Eq.2 . 


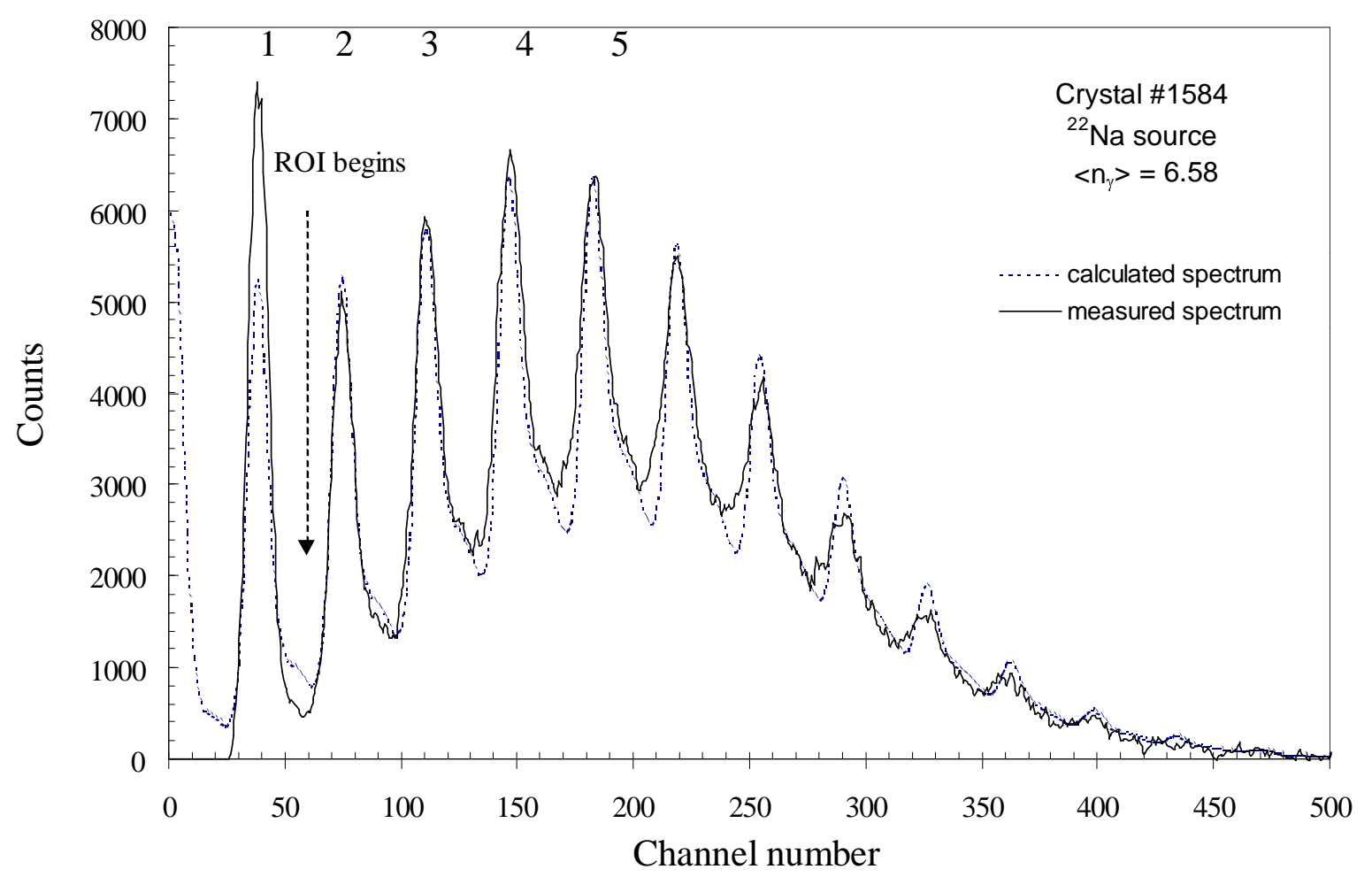

Fig. 11a: ${ }^{22} \mathrm{Na}$ spectrum of crystal \#1584 compared with its fit. The Region Of Interest was chosen in order to not account in the simulation of the low energy backgrounds.

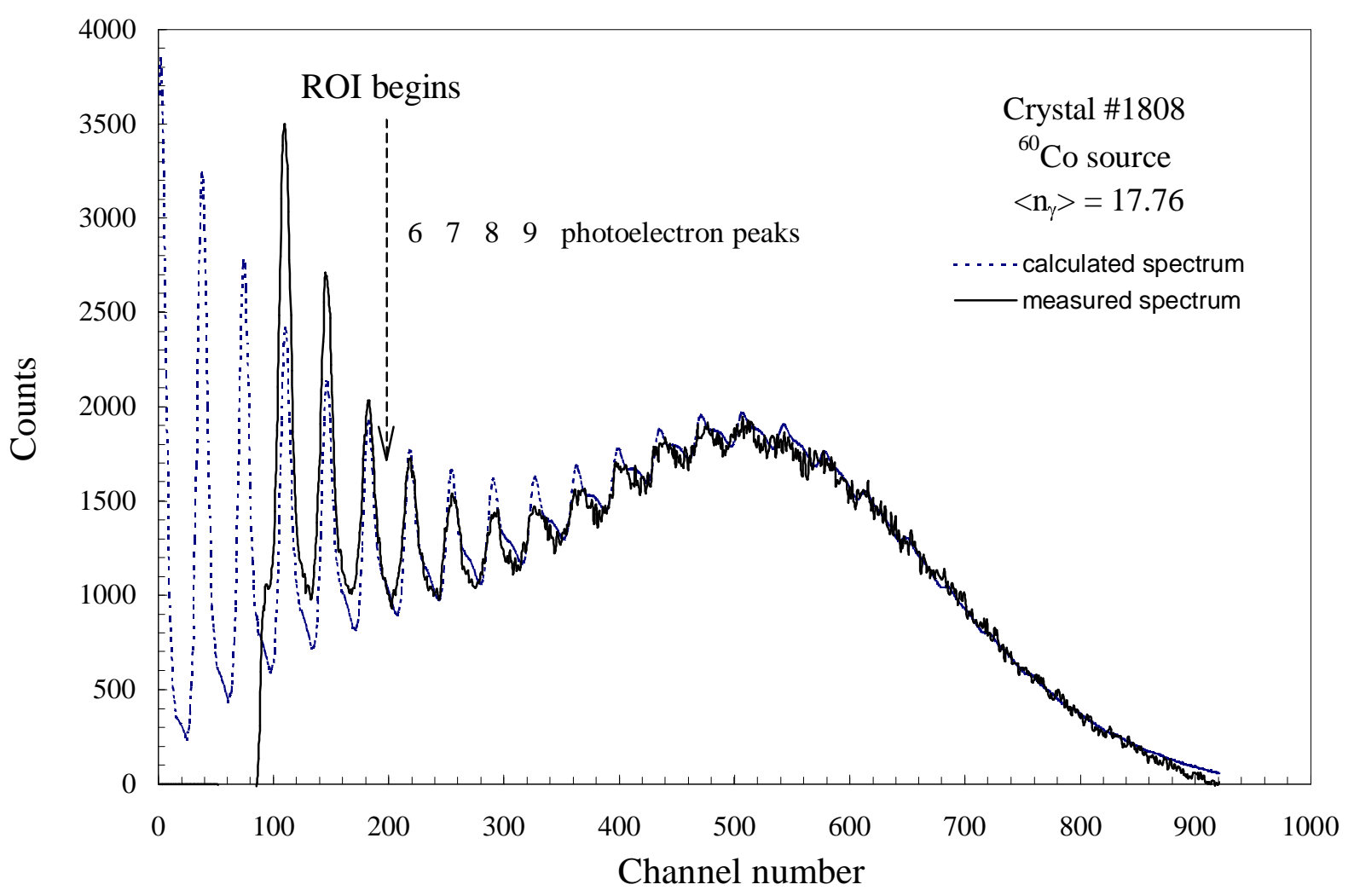

Fig. 11b: ${ }^{60}$ Co spectrum of crystal \#1808 compared with its fit. The Region Of Interest was chosen in order to not account in the simulation of the low energy backgrounds. 


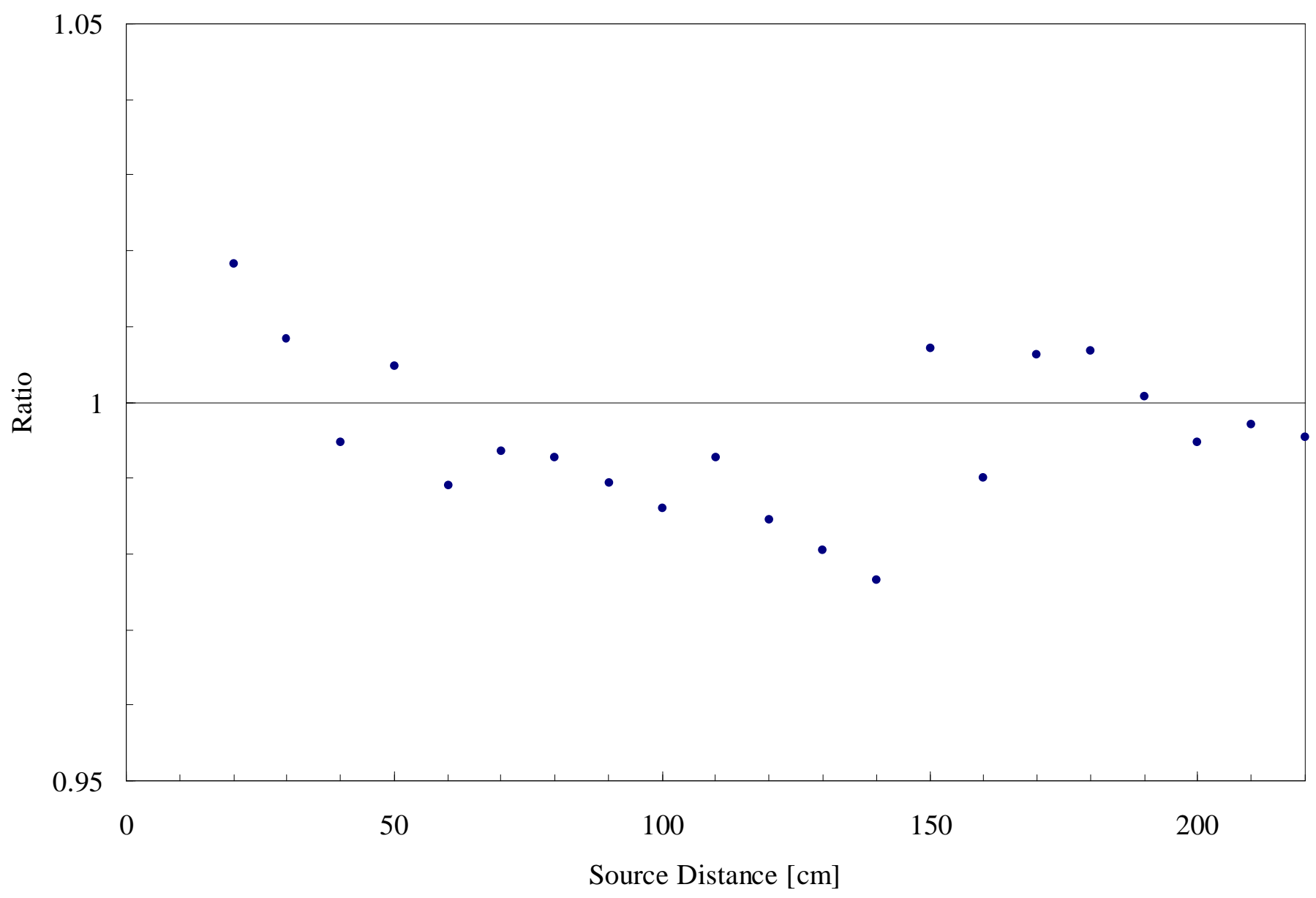

Fig. 12 : Ratio between ${ }^{60} \mathrm{Co}$ scan and ${ }^{22} \mathrm{Na}$ tagged scan with the same crystal (\#1858). 